\title{
Phase Structure and Mechanical Property of Blends of Organosolv Lignin Alkyl Esters with Poly( $\varepsilon$-caprolactone)
}

\author{
By Yoshikuni TERAMOTO, ${ }^{\dagger, *}$ Seung-Hwan LEE, and Takashi ENDO
}

Organosolv lignin (OSL) alkyl esters (carbon number $n, 2-5)$ were synthesized by homogeneous reaction with different acyl anhydrides/4-dimethylamino pyridine in pyridine solution. Miscibility of the esterified OSL with poly( $\varepsilon$-caprolactone) (PCL) was investigated through DSC, morphological observation, and spectroscopy. DSC revealed a composition-dependent shift in $T_{\mathrm{g}}$ for blends of OSL esters $(n=3-5)$ with PCL; thus these three systems can be regarded as miscible on a $T_{\mathrm{g}}$-detection scale. AFM indicated that no remarkable phase separation occurred in these blends. In observations of crystallization behavior of the PCL component, the OSL derivatives of $n=4$ and 5, the alkyl ester substituents of which make a higher similarity in chemical structure with a repeating unit of PCL, exhibited a higher level of miscibility with the aliphatic polyester, compared to the other OSL derivative of $n=3$. Thermal-molded blend sheets of OSL esters of $n=3-5$ with PCL attained $\geq 500 \%$ of elongation at rupture at $20^{\circ} \mathrm{C}$ even when the blends contained $50 \mathrm{wt} \%$ of the respective esterified lignin components, reflecting the good miscibility of these OSL esters with PCL.

KEY WORDS: Organosolv Lignin / Alkyl Ester / Poly( $\varepsilon$-caprolactone) / Blends / Miscibility / Glass Transition / Isothermal

Crystallization /

Currently, studies on bioethanol production from lignocellulosics are being actively pursued. ${ }^{1-4}$ Irrespective of the bioethanol manufacturing process, lignin is inevitably produced as a consequence of the saccharification of the cellulosic components of lignocellulosics. Although the lignin by-product is considered merely as a heat resource for the process in the present circumstances, its effective application will contribute to cost reduction of the total system for biofuel production.

Generally, however, the utilization of lignin as a solid material is constrained by poor film formability and less thermal moldability due to its molecular architecture and intermolecular interactions in itself. Upon heating, lignin molecules decompose rather than soften and flow. In order to alter lignin's viscoelastic properties, considerable efforts have been conducted to employ lignin components either as extenders in polyurethanes, phenol-formaldehyde resins, epoxies, and acrylics, or as grafting backbones for the attachment of other synthetic polymer chains. ${ }^{5}$

Meanwhile, the importance of polymer blends is now well established as an alternative method of developing completely novel polymers. Furthermore, polymer blending can offer opportunities to modify the thermal and other physical properties of the target polymers. However, it is generally difficult to prepare miscible blends due to the small contribution of the mixing entropy and therefore, in most cases, certain attractive intermolecular interactions between the component polymers should be adopted in order to attain the miscibility or practically good compatibility. Lignin blends or other combinations with synthetic polymers have been investigated many times over the last three decades ${ }^{6-8}$ for the purpose of utilizing woody biomass as some significant material. Recently, a few research groups focused on intermolecular interactions to obtain lignin-based polymer blends; e.g., ionic interaction, ${ }^{9}$ hydrogen-bonding attraction, ${ }^{10-15}$ and a specific structure of the counterpart polymer ${ }^{16-18}$ were utilized as driving forces to enhance the miscibility or compatibility of two constituent polymers. Most recently, miscibility of modified lignins was described systematically by using a solubility theory. ${ }^{19}$

Aliphatic polyesters, including bacterially produced poly(hydroxyalkanoate)s, have attracted considerable interest as candidate materials that could provide good biocompatibility and environmental degradability. Ghosh and Glasser have reported on the blends of organosolv lignin (OSL) and its esters with poly(3-hydroxybutyrate) (PHB) and revealed that OSL and its butyrate have a high degree of compatibility, and that lignin inhibits or retards PHB-crystallization. ${ }^{20} \mathrm{Li}$ and Sarkanen have reported on the miscibility and mechanical property of alkylated and acylated kraft lignins with aliphatic polyesters. ${ }^{17,18}$ They found that the key to forming miscible blends for ethylated and methylated kraft lignins was methylene/carboxylate group ratios $\left(\mathrm{CH}_{2} / \mathrm{COO}\right.$ 's) of the counterpart aliphatic main-chain polyesters. The interactions between the alkylated kraft lignin components and polyester chains were most favorable when the $\mathrm{CH}_{2} / \mathrm{COO}$ ratio was between 2.5 and 3.0. These studies indicate that a simple derivatization of lignins offers opportunities to obtain the hyperfine multicompositions with aliphatic polymers even though the lignins are an aromatic-rich polymer.

In the present study, we synthesized OSL acetate (OSL-Ac), propionate (OSL-Pr), butyrate (OSL-Bu), and valerate (OSL-

National Institute of Advanced Industrial Science and Technology (AIST), Biomass Technology Research Center (BTRC), 2-2-2, Hirosuehiro, Kure 7370197, Japan

†Present address: Division of Forest and Biomaterials Science, Graduate School of Agriculture, Kyoto University, Sakyo-ku, Kyoto 606-8502, Japan *To whom correspondence should be addressed (Tel: +81-75-753-6252, Fax: +81-75-753-6300, E-mail: termamoto@kais.kyoto-u.ac.jp). 
Va). In previous papers concerning a sulfuric acid-free ethanol cooking pretreatment for enzymatic hydrolysis of woody biomass, ${ }^{3,4}$ we demonstrated that a lignin by-product analogous to OSL were obtained and soluble in common organic solvents. Therefore, to explore an improved utilization opportunity of our lignin by-product, the esterified samples of commercial OSL were selected as a blending ingredient. A representative aliphatic polyester, poly( $\varepsilon$-caprolactone) (PCL), was chosen as the aliphatic polyester counterpart. If we can design an adequate miscible blend system, a substantial amount of lignins should be incorporated into thermal moldable solid materials without diminishing the mechanical property and the biodegradability of PCL. Moreover, or rather, the resulting blend system can be a biodegradation-controllable material of great promise based on a difference in the degradation behavior between the constituents. In the present study, we investigated an effect of the side-chain length of the OSL ester component on the miscibility of a series of OSL ester/PCL blends through differential scanning calorimetry (DSC) analysis, atomic force microscopy (AFM) observation, and FT-IR and solid-state NMR spectroscopy. Tensile property of the blend series was also examined for film specimens made by thermal molding. In general, the tensile mechanical strength, vapor absorption property, biodegradability, etc. of polymer materials should be considerably affected by the development of crystallinity. Therefore, isothermal melt-crystallization experiments were also carried out, and the morphology observed by optical microscopy is discussed in relation to the blend miscibility.

\section{EXPERIMENTAL}

\section{Materials}

Organosolv lignin (OSL) was purchased from Aldrich Chemical Co. Poly( $\varepsilon$-caprolactone) (PCL) was purchased from Wako Pure Chemical Industries, Ltd.; its nominal molecular weight was 40,000. Butyric and valeric anhydrides were obtained from Aldrich Chemical Co. Other solvents and chemicals used in this study were purchased from Wako Pure Chemical Industries, Ltd.; these were all of guaranteed reagent grade and were used without further purification.

\section{Esterification of OSL}

OSL ( $3 \mathrm{~g}$ ) was esterified at $100{ }^{\circ} \mathrm{C}$ with $45 \mathrm{~mL}$ of an acid anhydride appropriate for the product in $30 \mathrm{~mL}$ of pyridine containing $1.5 \mathrm{~g}$ of 4-dimethylaminopyridine. After a homogeneous reaction for $3 \mathrm{~h}$, the esterified products (OSL acetate (OSL-Ac), propionate (OSL-Pr), butyrate (OSL-Bu), and valerate (OSL-Va)) were recovered by precipitation in water (OSL-Ac and OSL-Pr) or diethyl ether (OSL-Bu and OSL-Va) and then washed with the respective precipitating media. Then, the esterified products were dried at $40^{\circ} \mathrm{C}$ in vacuo for $48 \mathrm{~h}$.

\section{Characterization of OSL and its Esters}

Methoxyl $\left(-\mathrm{OCH}_{3}\right)$ group content in OSL was determined by a method of Viebock and Schwappach. ${ }^{21}$ Elemental analysis was conducted at Center for Organic Elemental Microanalysis in Kyoto University. The phenyl propane unit (PPU) formulae of the OSL used could be determined to be $\mathrm{C}_{9} \mathrm{H}_{8.7} \mathrm{O}_{2.6^{-}}$ $\left(\mathrm{OCH}_{3}\right)_{0.83}$. The PPU molecular weight of the OSL was 184 $\mathrm{g} / \mathrm{mol}$.

Functional group contents of OSL and the degree of esterification were further determined by FT-IR and ${ }^{1} \mathrm{H}$ NMR spectroscopy. FT-IR spectra were obtained by using a PerkinElmer Spectrum GX spectrometer. A standard $\mathrm{KBr}-$ pellet method was employed for all the measurements. $300 \mathrm{MHz}{ }^{1} \mathrm{H}$ NMR spectra were measured at $20^{\circ} \mathrm{C}$ for OSL and OSL esters by using a Varian INOVA 300 apparatus. A pulse width of $3.0 \mu$ s was used and 256 scans were conducted. Approximately $100 \mathrm{mg}$ of OSL or OSL esters was dissolved in $1 \mathrm{~mL}$ DMSO- $d_{6}$ (99.9\% deuterated, $0.05 \%$ tetramethylsilane, Cambridge Isotope Laboratories, Inc.) and left overnight over molecular sieves to reduce water contamination. Chemical shift assignments were taken from the literature. ${ }^{19,22-25}$ Functional groups present in OSL were detemined by ${ }^{1} \mathrm{H}$ NMR spectroscopy using dichloromethane (DCM) as internal reference. Aromatic protons (ArH, 8-6.2 ppm) were used as a reference. Interference occurred between aromatic alcohol (ArOH) and aldehyde $(\mathrm{CHO})$ proton signals in the $8-11 \mathrm{ppm}$ range. After getting the initial spectrum of OSL in DMSO- $d_{6}$, by an addition of $20 \mathrm{vol} \% \mathrm{D}_{2} \mathrm{O}$, an exchange of the $\mathrm{ArOH}$ protons with a deuterium nucleus of $\mathrm{D}_{2} \mathrm{O}$ occurred, whereas the $\mathrm{CHO}$ protons were not substituted by deutrium. ${ }^{19,25}$ Therefore, a subtraction of the signal with $\mathrm{D}_{2} \mathrm{O}$ from the signal with the plain DMSO- $d_{6}$ canceled out the $\mathrm{CHO}$ proton contribution, then the ArOH content could be estimated. Aliphatic alcohol $(\mathrm{AlOH})$ content in OSL was calculated from the total and aromatic hydroxyl conversions of acetylated OSL. The numbers of functional groups (per PPU) of OSL are listed in Table I. The extent of esterification of the respective OSL esters was determined from the ester protons, as shown late.

The molecular weights of OSL and its esters were determined by using a TOSOH HLC-8220 gel permeation chromatograph equipped with a refractive index detector and two TSK-GEL SuperAWM-H columns connected with each other. The measurement was conducted by using $N$-methylpyrrolidone containing $10 \mathrm{mM} \mathrm{LiBr}$ as the mobile phase at a flow rate of $0.3 \mathrm{~mL} / \mathrm{min}$. The concentration of the test sample was $1.0 \mathrm{~g} / \mathrm{L}$ and the quantity of injection was $20 \mu \mathrm{L}$. The system was calibrated with monodisperse polystyrene standards.

\section{Preparation of Blend Samples}

OSL ester/PCL polymer blends were prepared in film form from mixed polymer solutions by solvent evaporation. Chloroform was selected as a common solvent. $1.0 \mathrm{wt} \%$ solutions of

Table I. Characterization of OSL used in this work

\begin{tabular}{|c|c|c|c|c|c|c|c|}
\hline \multirow[b]{2}{*}{ Sample } & \multicolumn{5}{|c|}{ Functional groups per PPU } & \multirow[b]{2}{*}{$10^{-3} M_{\mathrm{w}}$} & \multirow[b]{2}{*}{$M_{\mathrm{w}} / M_{\mathrm{n}}$} \\
\hline & $\begin{array}{c}\text { total } \\
\mathrm{OH}\end{array}$ & $\mathrm{AlOH}$ & $\mathrm{ArOH}$ & $-\mathrm{OCH}_{3}$ & $\mathrm{ArH}$ & & \\
\hline OSL & 1.23 & 0.61 & 0.62 & 0.83 & 2.92 & 3.38 & 3.52 \\
\hline
\end{tabular}


OSL ester and PCL were prepared separately and mixed with each other in the desired proportions (OSL ester/PCL $=100 /$ $0,95 / 5,90 / 10,85 / 15,80 / 20,75 / 25,70 / 30,65 / 35,60 / 40$, $50 / 50,40 / 60,30 / 70,20 / 80,10 / 90$, and $0 / 100$ in weight). After stirring over a time period of $24 \mathrm{~h}$ at room temperature $\left(25^{\circ} \mathrm{C}\right)$, each mixed solution (transparent but brown) was poured into a Teflon tray and a film sheet was prepared by solvent evaporation at $25^{\circ} \mathrm{C}$ and atmospheric pressure. Before conducting DSC measurements, the as-cast film samples were dried at $40^{\circ} \mathrm{C}$ in vacuo for $12 \mathrm{~h}$.

\section{Characterization of Blend Samples}

Phase morphology was observed using a PSIA atomic force microscope (AFM) XE-100. In order to prepare the test specimens, OSL or its ester derivatives and PCL were first dissolved in THF. The total polymer concentration was regulated at $1 \mathrm{wt} \%$ and the blending ratio of two polymers was 50/50 in weight. Thin films of the polymer blends were prepared by placing a droplet of the mixed solution onto a silicon wafer and spinning at $2500 \mathrm{rpm}$ at $20^{\circ} \mathrm{C}$ for $45 \mathrm{~s}$, then conditioned at $20^{\circ} \mathrm{C}$ for $48 \mathrm{~h}$. The phase morphology of the thin films was visualized in a true non-contact mode of AFM. To record a phase image, the phase lag of the cantilever vibration compared to the $\mathrm{z}$-piezo drive voltage was monitored as the probe scanned surface with a preset constant amplitude of vibration.

Wide-angle X-ray diffraction (WAXD) measurements were carried out by using an RINT-TTR III diffractometer (Rigaku Corporation, Tokyo, Japan). Nickel-filtered $\mathrm{CuK}_{\alpha}$ radiation $(\lambda=0.1542 \mathrm{~nm})$ was used at $50 \mathrm{kV}$ and $30 \mathrm{~mA}$. A disk pellet (diameter, $1 \mathrm{~cm}$; thickness, $1 \mathrm{~mm}$; and weight, $0.2 \mathrm{~g}$ ) was prepared for the measurements by using a disk pellet preparation kit for infra-red spectroscopy. The diffraction intensity was measured in the range of $2 \theta=4-45^{\circ}$.

DSC was performed by using a PerkinElmer DSC7. The measurements were carried out on 5-mg samples under a nitrogen atmosphere after calibrating the temperature readings with an indium standard. The samples were first heated from $-150{ }^{\circ} \mathrm{C}$ to $220^{\circ} \mathrm{C}$ at a scanning rate of $20^{\circ} \mathrm{C} / \mathrm{min}$ (first heating scan) and then immediately quenched to $-150{ }^{\circ} \mathrm{C}$ at a rate of $500^{\circ} \mathrm{C} / \mathrm{min}$. The second heating scans were run from $-150{ }^{\circ} \mathrm{C}$ to $220^{\circ} \mathrm{C}$ at a scanning rate of $20^{\circ} \mathrm{C} / \mathrm{min}$ to record stable thermograms. For estimation of the glass transition temperature $\left(T_{\mathrm{g}}\right)$ of each sample, the midpoint of the discontinuity in heat flow was recorded. The melting temperature $\left(T_{\mathrm{m}}\right)$ and the apparent enthalpy of fusion $\left(\Delta H_{\mathrm{f}}\right)$ were determined from the maximum and the area of the melting endotherm, respectively.

The crystallization behavior was also examined by following the time-coursing spherulitic growth under a polarizing optical microscope (POM). An Olympus BX50 polarizing microscope equipped with a Mettler FP82HT/FP90 hot-stage device was used for the purpose. The spherulitic growth was monitored by the following procedure. A small piece of blend film with the 50/50 composition, weighing about $0.3 \mathrm{mg}$, was sandwiched between the slide and cover glasses. Prior to the initiation of the crystallization process, the respective samples were heated to $150{ }^{\circ} \mathrm{C}$ and, subsequently, they were quickly transferred to the hot-stage thermo-regulated at the desired isothermal crystallization temperature $T_{\mathrm{ic}}$. The grown spherulites were observed by taking photomicrographs at appropriate intervals of time.

FT-IR spectroscopy was performed in a manner similar to that in the characterization of OSL and its esters.

The $400 \mathrm{MHz}{ }^{13} \mathrm{C} \mathrm{CP} / \mathrm{MAS}$ solid-state NMR spectra were measured by using a Varian Unity INOVA 400WB NMR apparatus. All the measurements were carried out at $20^{\circ} \mathrm{C}$ under the following conditions: pulse width, $4.0 \mu \mathrm{s}$; scan number, 2048; MAS speed, $5 \mathrm{kHz}$; contact time, $1.0 \mathrm{~ms}$.

Film sheets of blends for tensile tests were prepared by using a Toyo-Seiki hot-pressing apparatus. For the molding at $150^{\circ} \mathrm{C}$, pressure was applied to each molten blend gradually to reach $5 \mathrm{MPa}$ in $3 \mathrm{~min}$; subsequently, it was increased quickly to $15 \mathrm{MPa}$, and this application was maintained for $30 \mathrm{~s}$. Following this, as soon as the pressure was released, the sample was transferred to another compressing apparatus and quickly coldpressed at $25^{\circ} \mathrm{C}$ and $15 \mathrm{MPa}$ for $2 \mathrm{~min}$. After being released again from the compressed state, the molded polymer sheet was finally conditioned at $20^{\circ} \mathrm{C}$ and $60 \% \mathrm{RH}$ for $48 \mathrm{~h}$. Specimens were cut from the sheet to 3 dumbbell-type test pieces (JIS K6251: length and width of the narrow portion, $20 \mathrm{~mm}$ and $5 \mathrm{~mm}$, respectively) with a thickness of $0.4 \mathrm{~mm}$. Tensile behavior was examined for these specimens at $20^{\circ} \mathrm{C}$ by using a Shimadzu Autograph AGS-5kNG. The strain rate and span length were $0.5 \mathrm{~mm} / \mathrm{min}$ and $40 \mathrm{~mm}$, respectively. Three specimens of each blend sample were employed for the measurement and the averaged data were adopted.

\section{RESULTS AND DISCUSSION}

\section{Characterization of OSL Esters}

As shown in Figure 1, FT-IR measurements demonstrated that the bands of hydroxyls for each ester derivative disappeared, revealing that the respective OSL esters are fully substituted ones. The extent of esterification was estimated by ${ }^{1} \mathrm{H}$ NMR spectroscopy (Figure 2). For this estimation, the already mentioned $\mathrm{ArH}$ (8-6.2 ppm) and $\mathrm{ArOH}$ (11-8 ppm) signals (see Experimental section), and besides the ester signals listed below were used: OSL-Ac, $\delta=2.5-2.1$ (aromatic $-\mathrm{CH}_{3}$ ), 2.1-1.6 (aliphatic $-\mathrm{CH}_{3}$ ); OSL-Pr, aromatic $\delta=1.3-1.1$ (aromatic $\left.-\mathrm{CH}_{3}\right), 1.1-0.5$ (aliphatic $\left.-\mathrm{CH}_{3}\right) ; 2.9$ 2.4 (aromatic $-\mathrm{CH}_{2}-$ ), 2.4-1.8 (aliphatic $-\mathrm{CH}_{2}-$ ); OSL-Bu, $1.1-0.5\left(-\mathrm{CH}_{3}\right), 1.8-1.25\left(\beta-\mathrm{CH}_{2}-\right), 2.4-2.0\left(\alpha-\mathrm{CH}_{2}-\right)$; OSLVa, 1.0-0.4 $\left(-\mathrm{CH}_{3}\right), 1.8-1.0\left(\gamma-\mathrm{CH}_{2}-\right.$ and $\left.\beta-\mathrm{CH}_{2}-\right), 2.4-2.0$ $\left(\alpha-\mathrm{CH}_{2}-\right)$. As a result, the conversion of total hydroxyl groups in the original OSL was as follows: $100 \%$ for OSL-Ac and OSL-Pr, $84 \%$ for OSL-Bu, and $90 \%$ for OSL-Va, each evaluated relative to $\mathrm{ArH}$ protons as reference. Even though FT-IR confirmed the disappearance of $\mathrm{OH}$ groups for the OSL esters, the apparent conversion did not attain $100 \%$ for OSL-Bu and OSL-Va. It is fairly assumed that the OSL-Ac and OSL-Pr could be recovered successfully by the precipitation in water 


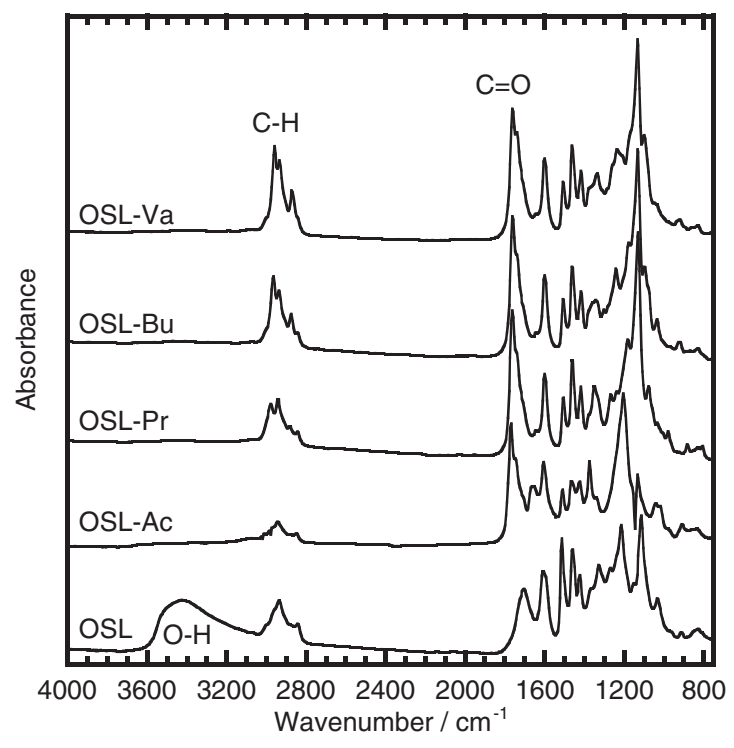

Figure 1. FT-IR spectra of OSL and OSL esters.
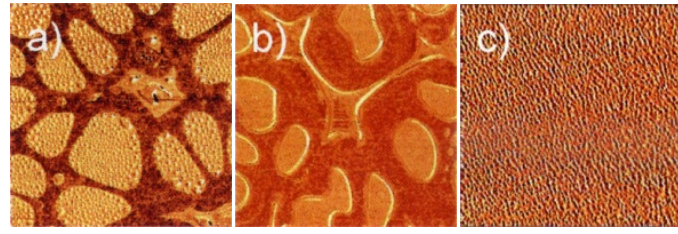

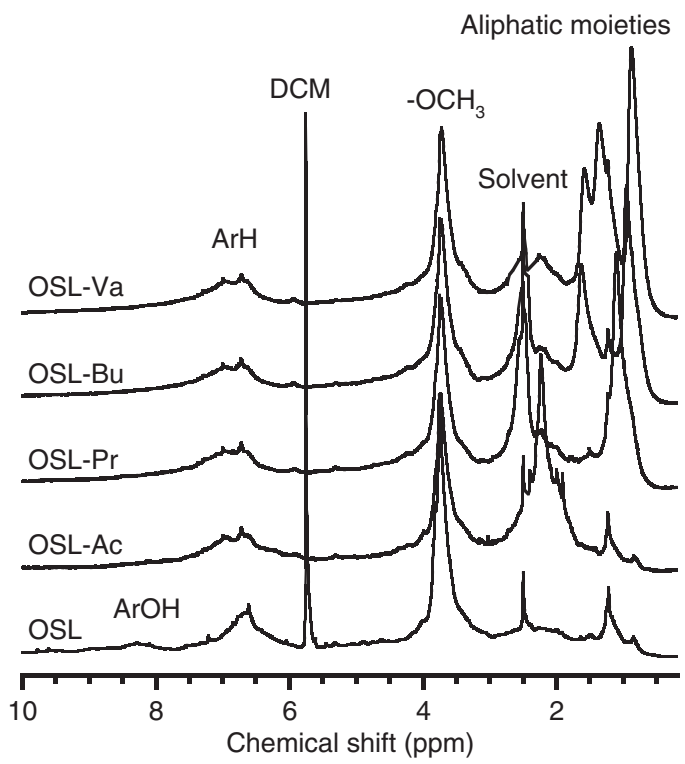

Figure 2. ${ }^{1} \mathrm{H}$ NMR spectra of OSL and OSL esters.

Figure 3. AFM images $\left(20 \times 20 \mu \mathrm{m}^{2}\right)$ of $50 / 50$ blends of (a) OSL, (b) OSL-Ac, (c) OSL-Pr, (d) OSL-Bu, and (e) OSL-Va with PCL.

after the esterification. In contrast, it is very likely to be difficult to completely recover the OSL-Bu and OSL-Va in the step of purification because the precipitation was performed in diethyl ether. The unrecovered fraction soluble in diethyl ether should be rich in ester moieties for these OSL esters.

The weight-average molecular weight of the respective OSL esters was determined by GPC: $3.36 \times 10^{3}$ (OSL), $3.40 \times 10^{3}$ (OSL-Ac), $3.43 \times 10^{3}$ (OSL-Pr), $4.30 \times 10^{3}$ (OSL-Bu), and $4.75 \times 10^{3}(\mathrm{OSL}-\mathrm{Va})$, the value increasing with the carbon number $n$ of the introduced acyl unit. The relatively higher molecular weights for the OSL-Bu and OSL-Va indicate loss of a lower molecular weight fraction by purification with diethyl ether. The molecular weight distributions for the four products were 3.52 (OSL), 3.99 (OSL-Ac), 3.20 (OSL-Pr), 3.19 (OSL$\mathrm{Bu}$ ), and 3.24 (OSL-Va).

These OSL esters were used for the preparation of blends with PCL. The as-cast films of the OSL ester/PCL blends were visually homogeneous, but brown in color.

\section{Phase-detection AFM Images}

AFM provides useful information about the morphology in the bulk surface of polymer blends. In particular, phasedetection imaging AFM can be used to characterize both chemical and morphological organization. By this technique, it is possible to visualize a surface region where one constituent is enriched, even when this enrichment creates no identifiable topographical feature.

Figure 3 illustrates phase-detection AFM images $(20 \times 20$ $\mu \mathrm{m}^{2}$ ) of the surface of films of OSL/PCL and OSL ester/PCL blends (50/50 composition in weight); these films were allowed to stand for $48 \mathrm{~h}$ at $25^{\circ} \mathrm{C}$ after spin-coating on silicon wafers. Figure $3 a$ and $3 b$ display the image of OSL/PCL and that of OSL-Ac/PCL, respectively, both indicating a phase separation feature of the respective surfaces. The scale of the phase separation was estimated to be from $c a$. 5-10 $\mu \mathrm{m}$ for the OSL/PCL and 3-6 $\mu \mathrm{m}$ for the OSL-Ac/PCL sample. On the other hand, the blends of OSL-Pr, OSL-Bu, and OSL-Va with PCL exhibited no apparent phase separation, as shown in Figure 3c, 3d, and 3e. The line-like clefts observed for the blends of OSL-Bu and OSL-Va reflect the impingement of PCL spherulites, which will be discussed later. Figure 4 shows fine images $\left(2 \times 2 \mu \mathrm{m}^{2}\right)$ of those three blend samples. Again, these images provide no appreciable phase separation even at a sub-micron order, implying improvement of the compatibility of the OSL derivatives with PCL due to esterification. For other compositions than 50/50, phase separation was observed again for OSL and OSL-Ac blends, whereas no apparent phase separation was detected for the other three blends.

\section{Effect of Side-chain Length on Thermal Transition Behav- ior}

The polymer-polymer miscibility is commonly estimated by 

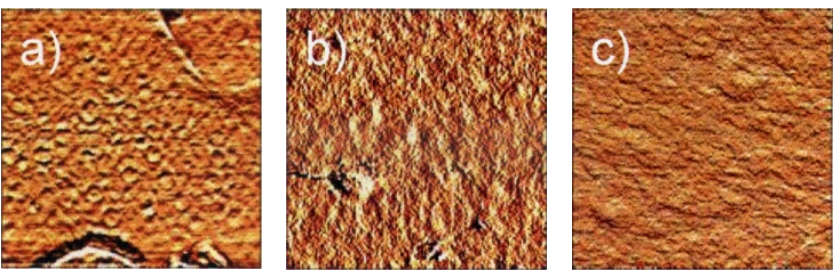

Figure 4. AFM images $\left(2 \times 2 \mu \mathrm{m}^{2}\right)$ of $50 / 50$ blends of (a) OSL-Pr, (b) OSL$\mathrm{Bu}$, and (c) OSL-Va with PCL.

the determination of the glass transition temperature $\left(T_{\mathrm{g}}\right)$ of the blends. If any sample of a binary polymer system exhibits a single glass transition between the $T_{\mathrm{g}}$ values of both components and a composition-dependent shift in $T_{\mathrm{g}}$ of the blend is observed, then the system can be regarded as a highly miscible one on a $T_{\mathrm{g}}$-detection scale that is usually assumed to be less than a couple of tens of nanometers. ${ }^{26-28}$

Figure 5a illustrates DSC thermograms obtained for a series of blends of OSL-Va/PCL. The PCL homopolymer exhibits a sharp melting endotherm with a peak maximum at $60{ }^{\circ} \mathrm{C}$ and a small base-line gap below $-50{ }^{\circ} \mathrm{C}$, which is caused by passing through the glass transition point of the polymer. From a precise reading of the midpoint of the discontinuity in heat flow, the $T_{\mathrm{g}}$ of the PCL was found to be $-65^{\circ} \mathrm{C}$ (Figure 5a, 0/ 100). The other component in this mixture, OSL-Va, was highly amorphous and exhibited a heat-flow discontinuity whose midpoint was estimated to be $40^{\circ} \mathrm{C}$ (Figure 5a, 100/0). For the blends of the two polymers, a single glass transition was detected for all of the compositions investigated (10/90$95 / 5$ ), with their $T_{\mathrm{g}} \mathrm{s}$ varying between the $T_{\mathrm{g}}$ values of the two components according to the composition. This demonstrates that the polymer pair is miscible in the amorphous fraction of the mixture. In the composition range of OSL-Va/PCL $=0$ / 100-60/40, the degree of the $T_{\mathrm{g}}$ elevation with an increase in OSL-Va content was relatively small, whereas at OSL-Va-rich compositions, the blend $T_{\mathrm{g}}$ shifted more to the higher temperature side with increasing OSL-Va concentration. Wide-angle $\mathrm{X}$-ray diffraction profiles confirmed that an observed crystal structure was identical with that of PCL, concerning for the blends whose DSC thermograms exhibited endotherm at around $60^{\circ} \mathrm{C}$. The melting peak of PCL shifted slightly to lower temperatures as the OSL-Va concentration was increased up to $70 \mathrm{wt} \%$, and was no longer recognizable at a composition of $75 / 25$, as shown in Figure 5a. Such effects of a depression in the melting point and a repression in the development of crystallinity of the crystallizable component PCL, caused by the addition of the second component OSL-Va, are common features shared with other crystalline/amorphous polymer pairs that are capable of forming a miscible phase in their blends. $^{26-29}$ In Figure 5a, the exotherm appearing clearly in the DSC data for the five OSL-Va/PCL samples with the compositions of $65 / 35-30 / 70$ is due to the formation of PCL crystals that were induced during the heating scan; i.e., a coldcrystallization phenomenon took place after the onset of the glass transition of these samples on heating.
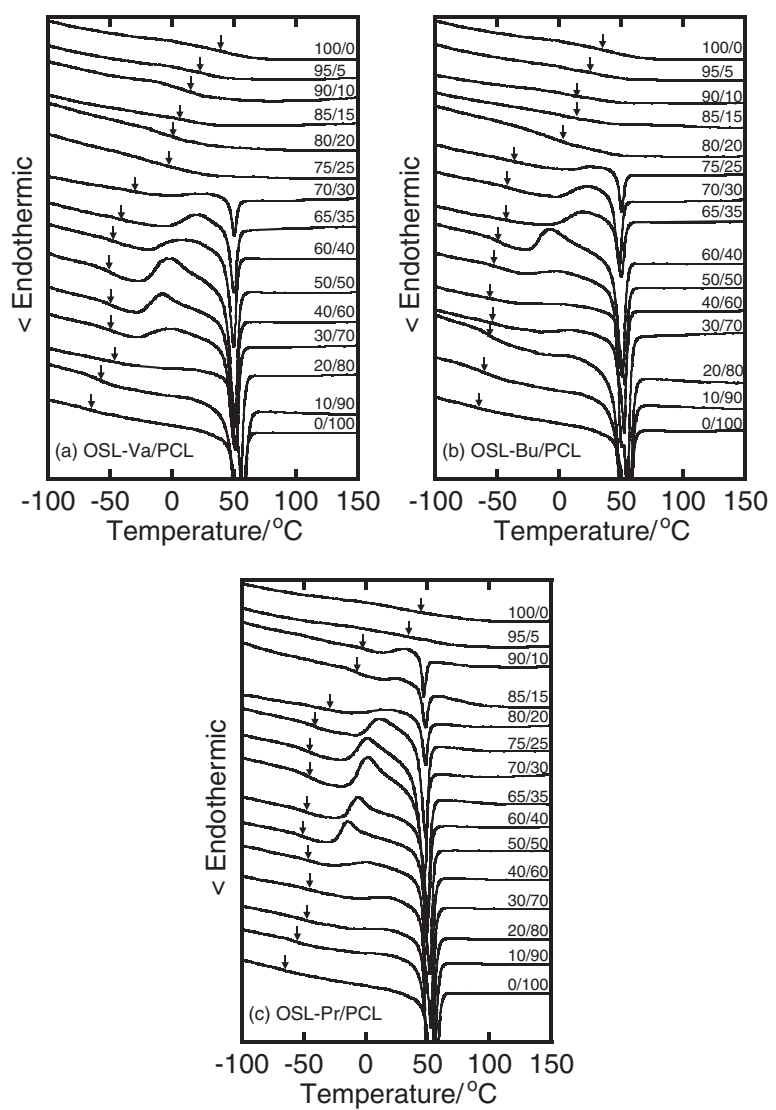

Figure 5. DSC thermograms (second heating scan) obtained for (a) OSL$\mathrm{Va} / \mathrm{PCL}$, (b) OSL-Bu/PCL, and (c) OSL-Pr/PCL blends. Arrows indicate $T_{\mathrm{g}}$ positions.

Figure 6 shows DSC thermograms obtained for OSL-Ac/ PCL blends. It is evident that the components of the polymer mixture are poorly miscible due to the less-significant shift in the $T_{\mathrm{g}} \mathrm{s}$ of both components. In particular, the compositions of 50/50-95/5 show two independent glass transitions. In addition, the melting endotherm of PCL is observable for every composition with the peak area reducing in direct proportion to the PCL content. Thus, the thermal properties of the respective polymer components are not affected by the presence of the other component in the OSL-Ac/PCL blends. This is in contrast to the case of the OSL-Va/PCL blends described above.

Figure 7 shows $T_{\mathrm{g}} v s$. composition plots for four series of blends of PCL with OSL alkyl esters $(n=2-5)$ with PCL. The miscibility characterization for these series should be significantly related with the side-chain length of the OSL esteres. The plots for OSL-Ac/PCL and OSL-Va/PCL blends in Figure 7 are based on the DSC data given in Figures 5a and 6, respectively. Blending PCL with OSL-Ac did not cause a shift in the $T_{\mathrm{g}}$ of the PCL components irrespective of the composition, and, therefore, this binary system is judged to be immiscible over the entire composition range. On the other hand, OSL-Bu and OSL-Pr showed miscibility with PCL in the thermal analysis, the DSC data being displayed in Figure 5b and $5 \mathrm{c}$, respectively. As shown in Figure 7, the OSL-Bu/PCL 


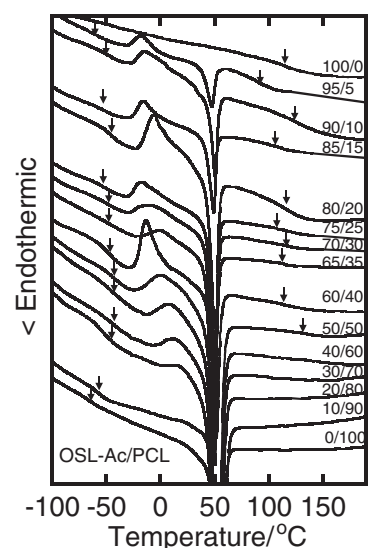

Figure 6. DSC thermograms (second heating scan) obtained for OSL-Ac/ PCL blends. Arrows indicate $T_{\mathrm{g}}$ positions.

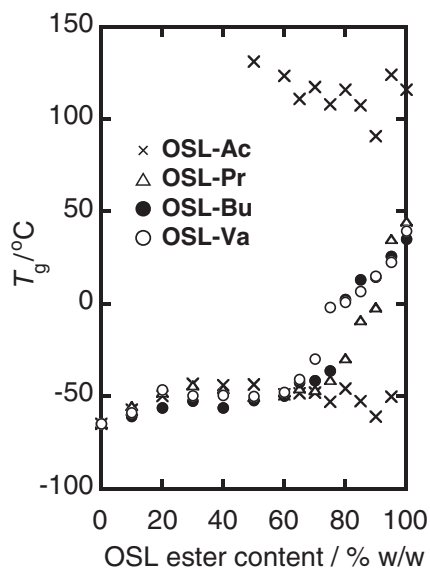

Figure 7. $T_{\mathrm{g}}$ vs. composition plots for blends of different OSL alkyl esters with PCL.

blends exhibit an appreciable elevation in PCL $T_{\mathrm{g}}$ at compositions containing $\geq 80 \mathrm{wt} \%$ OSL-Bu. This trend is similar to the $T_{\mathrm{g}}$ shift of the OSL-Va/PCL system. A $T_{\mathrm{g}}$ shift is also observed for the OSL-Pr/PCL blends, but it becomes prominent at compositions of $\geq 85 \mathrm{wt} \%$ OSL-Pr. Therefore, this blend system can be considered to attain a certain level of miscibility which would be inferior to the level of the OSL-Va/ PCL system.

Further inspection of the side-chain-length effect on the miscibility characteristics may be made from a viewpoint of the PCL crystallinity. In the OSL-Pr/PCL systems, the crystallization habit of PCL remained even at the 90/10 composition too rich in OSL-Pr (Figure 5c); thus fully amorphous blends were never obtained over the composition range of 90/10-10/90. In the OSL-Va/PCL and OSL-Bu/PCL systems, however, the crystallizability of PCL vanished completely when OSL-Va and OSL-Bu reached $75 \mathrm{wt} \%$ and $80 \mathrm{wt} \%$, respectively (Figure $5 \mathrm{a}$ and $5 \mathrm{~b}$ ). This observation supports the conclusion that OSL-Bu and OSL-Va show a higher degree of miscibility with PCL, compared with OSL-Pr.
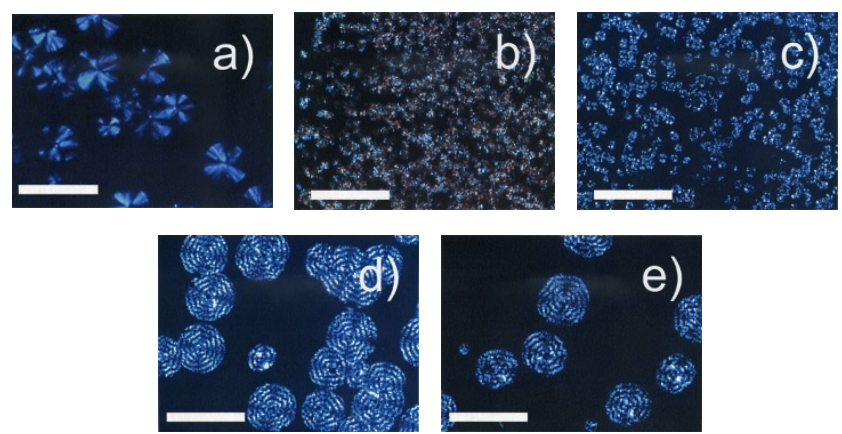

Figure 8. Polarized optical micrographs taken for plain PCL at $T_{\text {ic }}=40^{\circ} \mathrm{C}$ and $t=5 \mathrm{~min}$ and for $50 / 50$ blends of OSL ester/PCL series at $T_{\text {ic }}=35^{\circ} \mathrm{C}$ and $t=30 \mathrm{~min}$ : (a) plain PCL, (b) OSL-Ac/PCL, (c) OSL-Pr/PCL, (d) OSL-Bu/PCL, (e) OSL-Va/PCL. Scale bar denotes $100 \mu \mathrm{m}$.

\section{Spherulite Growth and Morphology}

For some of OSL ester/PCL blends allowing the crystal development of the PCL component, a spherulite growth was observed by polarized optical microscopy (POM). As a standard experiment, the blend samples were heated at $150{ }^{\circ} \mathrm{C}$ so that their thermal history was erased, and subsequently they were annealed isothermally at a crystallization temperature $T_{\mathrm{ic}}$ for time $t$.

Typical POM images obtained for 50/50 samples of the respective OSL ester/PCL series are collected in Figure 8, together with that for plain PCL. In the immiscible OSL-Ac/ PCL blend (see Figure 8b), the crystallization of the PCL component appeared to have proceeded in the originally segregated domain, but the total optical morphology was rather blear and lacked a definite sperulite texture. On the other hand, the spherulitic growth was clearly observed for the three blends of PCL with OSL-Pr, OSL-Bu, and OSL-Va, as shown in Figure $8 \mathrm{c}-8 \mathrm{e}$. During the growth, all the blends exhibited no explicit segregation of the non-crystallizable OSL ester component in both the intra-spherulitic region and the spherulitic contact region under the microscope. The spherulites ultimately impinged on one another and their apparent growth stopped then. For these three series of blends, therefore, it is natural to assume that the OSL ester fraction was incorporated in interlamellar regions within the spherulites.

Spherulites observed for the blends of PCL with the three OSL esters were all of negative birefringence, as judged from an optical retardation test with adequate crystalline plates. The blends of PCL with OSL-Bu and OSL-Va exhibited a so-called "Maltese cross" effect (Figure 8d and 8e). However, in the OSL-Pr/PCL blend, the spherulite texture was generally small and distorted, and worse in contrast (Figure 8c); such a nonuniform morphology may be ascribed to the relatively lower miscibility of this OSL ester with PCL.

A noteworthy observation is the pattern composed of periodic extinction rings, which appeared only in spherulites of the OSL-Bu/PCL and OSL-Va/PCL blends and never appeared in unblended PCL spherulites. As far as multicomponent polymer systems are concerned, this kind of banded extinction texture has been reported in several works on 

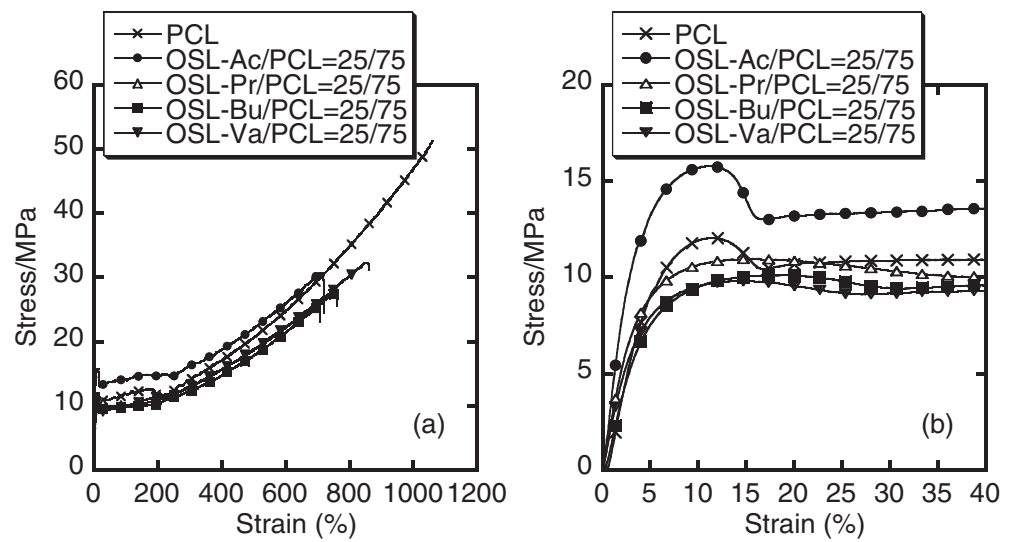

Figure 9. Stress-strain curves for plain PCL and 25/75 blends of OSL-Ac/PCL, OSL-Pr/PCL, OSL-Bu/PCL, and OSL-Va/PCL: (a) curves for the entire range; (b) shown on an enlarged scale for the initial stage of elongation.

miscible or partially miscible polymer blends, ${ }^{30-33}$ a few block copolymers, ${ }^{34,35}$ and a graft copolymer. ${ }^{36}$ Such a specific morphology has been interpreted by a twisted crystal model, in which the adsorption of impurity on crystal boundaries (i.e., growth faces and fold surfaces of lamellar crystals) allows the lamellae to twist around an axis of radial growth. ${ }^{31}$ The impurity involves an uncrystallizable polymeric diluent added to the crystallizable host polymer in the multicomponent systems. According to this model, OSL-Bu and OSL-Va can be taken as the non-crystalline polymer diluents to develop the extinction rings in the PCL spherulites.

\section{Driving Force for Attainment of Miscibility}

As has been described above, the miscibility characteristics are primarily related to the side-chain length of OSL esters. Usually, if a strong interaction such as hydrogen bonding and electrostatic ionic attraction prevails in a polymer blend, the shape and/or position of some of the spectroscopic signals of one or both components should change appreciably from their original situations. However, FT-IR spectra for miscible blends of OSL-Va/PCL and OSL-Bu/PCL with different compositions were found to show an essentially prorated mixture of two data for the constituent polymers. Further, ${ }^{13} \mathrm{C} \mathrm{CP} / \mathrm{MAS}$ NMR spectra measurements also revealed no appreciable interaction between the OSL alkyl ester and PCL components in the blends.

At the present stage, there is no definitive explanation for why the butyryl and valeryl substitutions are especially effective for the enhancement of miscibility in the binary system of the alkyl-esterified OSL/PCL mixture. However, the structural similarity appears to be an important factor; viz., OSL-Bu and OSL-Va have, most abundantly, the same structural unit as that of PCL (C-C-O-(CO)-C-C-C or C-O(CO)-C-C-C-C), if the aliphatic carbon atoms neighboring aliphatic hydroxyl groups are taken into account. In OSL-Pr, which contains the structure $\mathrm{O}-(\mathrm{CO})-\mathrm{C}-\mathrm{C}$, there is less frequency of occurrence of the same structural unit as that of PCL even when the aliphatic hydroxyl groups are esterified. This may be why more content of the OSL-Pr component was required for the shift in $T_{\mathrm{g}}$ of the blends, as shown in Figures $5 \mathrm{c}$ and 7. A similar explanation has been proposed to interpret a miscibility map conducted for the binary system of the alkyl-esterified cellulose/PCL mixture, where the carbon atoms $\mathrm{C}-1$ to $\mathrm{C}-6$ in a glucopyranose unit were considered to attain a structural unit similar to that of PCL. ${ }^{37-39}$ In the present system too, conceivably, the experimental results suggest that such a structural effect of the substituents is contributive to the attainment of miscibility, even though almost half the hydroxyl groups of the original OSL are aromatic ones (Table I).

\section{Mechanical Properties of Blends}

Film sheets of plain PCL and OSL ester/PCL blend (25/75, $50 / 50$, and $75 / 25$ ), which were obtained by melt-molding at $150{ }^{\circ} \mathrm{C}$ followed by quick cooling, were dark brown but homogeneous to the naked eye. Dumbbell-type specimens cut from the films were subjected to uniaxial stretching at $20^{\circ} \mathrm{C}$ to estimate the tensile properties of the blends. At the temperature, however, $75 / 25$ blend samples were generally so brittle, and thus the precise measurement was restricted to the other two compositions of $\geq 50 \mathrm{wt} \%$ PCL.

Figure 9 shows the stress-strain curves obtained for the original PCL and 25/75 blends of OSL ester/PCL. In these blends, actual lignin contents (before acylation) were 19.8 (OSL-Ac), 18.5 (OSL-Pr), 17.5 (OSL-Bu), and 16.5 wt \% (OSL-Va). Table II summarizes the resultant tensile parameters. Concerning the $25 / 75$ compositions, there appears to be a general tendency that the elongation at rupture remained $\geq 700 \%$. However, as can be seen in an enlarged illustration (Figure 9b), the yield stress of the immiscible OSL-Ac/PCL was higher than that of plain PCL, in contrast to the other three blends. The Young's moduli of all the blend samples were higher than that of plain PCL; the increment was remarkable for the immiscible OSL-Ac/PCL blend. Primarily, the increasing action of the Young's moduli was due to a brittle nature of the OSL esters. In the immiscible OSL-Ac/PCL system, this can be rather attributed to the facilitation of forming craze cracks including micro voids due to poor interfacial adhesion. Such craze cracks consist of oriented and molecularly drawn 

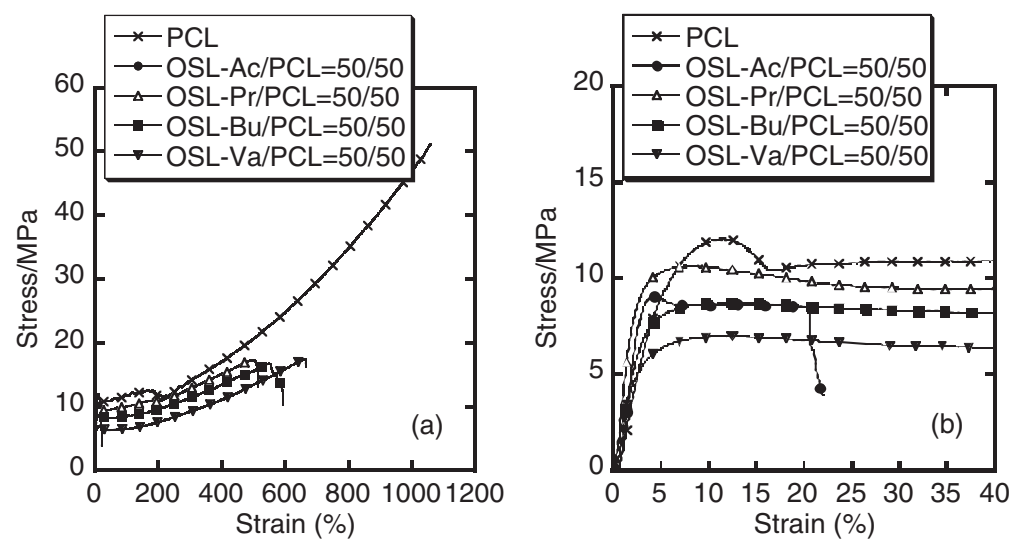

Figure 10. Stress-strain curves for plain PCL and 50/50 blends of OSL-Ac/PCL, OSL-Pr/PCL, OSL-Bu/PCL, and OSL-Va/PCL: (a) curves for the entire range; (b) shown on an enlarged scale for the initial stage of elongation.

Table II. Tensile property of OSL ester/PCL blends and plain PCL

\begin{tabular}{lrccc}
\hline & $\begin{array}{c}\text { Maximum } \\
\text { strength/MPa }\end{array}$ & $\begin{array}{c}\text { Elongation } \\
\text { at break }(\%)\end{array}$ & $\begin{array}{c}\text { Young's } \\
\text { modulus/MPa }\end{array}$ \\
\hline OSL-Ac/PCL $=25 / 75$ & 24.7 & 723 & 353 \\
& $50 / 50$ & 7.9 & 21 & 526 \\
OSL-Pr/PCL $=25 / 75$ & 25.8 & 710 & 281 \\
& $50 / 50$ & 15.5 & 516 & 430 \\
OSL-Bu/PCL $=25 / 75$ & 28.2 & 760 & 218 \\
& $50 / 50$ & 16.4 & 596 & 306 \\
OSL-Va/PCL $=$ & $25 / 75$ & 32.4 & 862 & 224 \\
& $50 / 50$ & 17.2 & 667 & 229 \\
plain PCL & & 51.3 & 1061 & 193 \\
\hline
\end{tabular}

parts which are not easily fractured. ${ }^{40}$ In an earlier stage of the stretching process, these oriented regions between the voids in the craze crack tend to prevent coalescence of the voids, which would result in a rigidity and catastrophic breakdown. Such craze cracks caused by phase separation seem to be rather less prevalent for the miscible pairs.

A further increase in the OSL ester content to $50 \mathrm{wt} \%$ in the blends (actual lignin contents before acylation; 39.6 (OSL-Ac), 37.1 (OSL-Pr), 35.1 (OSL-Bu), and $33.0 \mathrm{wt} \%$ (OSL-Va)) gave rise to a large different tensile behavior according to the blend miscibility. The immiscible OSL-Ac/PCL pair showed a striking decrease in elongation at rupture, while the elongation of the other three miscible blends remained $\geq 500 \%$, as shown in Figure 10. The decrease in ductility for the OSL-Ac/PCL blend can be attributed to poor interfacial adhesion in a micrometer scale. At this 50/50 composition, the formation of craze cracks was probably no longer effective in improving the stress transfer in the immiscible OSL-Ac/PCL blend, because there were too many interfacial regions. On the other hand, for the other three blend systems, such an interface region may be taken to be absent on a scale larger than ten nanometers. It is thus concluded that the miscible blend pairs acquire a practically feasible mechanical property even when the blends contain $50 \mathrm{wt} \%$ of the respective esterified lignin components.

\section{CONCLUSIONS}

Miscibility characterization was performed on blends of poly( $\varepsilon$-caprolactone) (PCL) with different organosolv lignin (OSL) fully-substituted in the alkyl ester form, the number $(n)$ of carbons in the acyl substituent ranging from 2 to 5 . Through thermal analysis by DSC, it was found that OSL butyrate (OSL-Bu) and valerate (OSL-Va) of $n=4$ and 5, respectively, exhibited high miscibility on blending with PCL. OSL propionate (OSL-Pr) of $n=3$ showed a certain degree of miscibility with PCL. AFM observations also showed that no remarkable phase separation occurred in these three blends. OSL acetate (OSL-Ac) of $n=2$ was completely immiscible with PCL. The three miscible blend pairs attained a practically feasible mechanical property even when the blends contained $50 \mathrm{wt} \%$ of the respective lignin ester components.

In the isothermal crystallization experiment by POM, a growth of sperulites having clearly banded extinction ring was observed for the blends containing OSL-Bu and OSL-Va. The blend pair of OSL-Pr/PCL also exhibited crystal growth in a spherulitic manner; however, the texture became distorted and worse in contrast. The better miscibility of the OSL-Bu/PCL and OSL-Va/PCL pairs was supported again by these optical observations of PCL melt-crystallization in the blends with different OSL esters.

For the attainment of better miscibility in the binary system of OSL alkyl ester and PCL, the structural similarity appears to be an important factor in the following respect: OSL-Bu and OSL-Va have, most abundantly, the same structural unit as that of PCL (C-C-O-(CO)-C-C-C or C-O-(CO)-C-C-C-C), if the aliphatic carbon atoms neighboring aliphatic hydroxyl groups are taken into account.

The present study indicated that a simple lignin derivatization offers opportunities to obtain hyperfine multicomposition with aliphatic polymers even though lignins are aromatic-rich polymers. The resulting blend systems can be a biodegradationcontrollable material of great promise. Concerning the present OSL ester/PCL blends, further characterization including nuclear magnetic relaxation, dynamic mechanical property, 
and detailed isothermal crystallization kinetics will be required, especially for more precise assessment of the mixing scale of the two polymer components.

Acknowledgment. The authors are deeply grateful to Ms. Naomi Kadotani, Ms. Noriko Tanaka, and Ms. Manami Asano (AIST) for their assistance in the experiments.

Received: November 20, 2008 Accepted: December 5, 2008 Published: January 21, 2009

\section{REFERENCES}

1. A. E. Farrell, R. J. Plevin, B. T. Turner, A. D. Jones, M. O'Hare, and D. M. Kammen, Science, 311, 506 (2006).

2. N. Mosier, C. Wyman, B. Dale, R. Elander, Y. Y. Lee, M. Holtzapple, and M. Ladisch, Bioresour. Technol., 96, 673 (2005).

3. Y. Teramoto, S. H. Lee, and T. Endo, Bioresour. Technol., 99, 8856 (2008).

4. Y. Teramoto, N. Tanaka, S. H. Lee, and T. Endo, Biotechnol. Bioeng., 99, 75 (2008).

5. "Lignin: Properties and Materials," W. G. Glasser and S. Sarkanen, Ed., American Chemical Society, Washington, DC, 1989.

6. S. L. Ciemniecki and W. G. Glasser, Polymer, 29, 1021 (1988).

7. S. L. Ciemniecki and W. G. Glasser, Polymer, 29, 1030 (1988).

8. D. Feldman, D. Banu, M. Lacasse, J. Wang, and C. Luchian, J. Macromol. Sci., Pure Appl. Chem., A32, 1613 (1995).

9. D. Hasegawa, Y. Teramoto, and Y. Nishio, J. Wood Sci., 54, 143 (2008).

10. J. F. Kadla and S. Kubo, Macromolecules, 36, 7803 (2003).

11. S. Kubo and J. F. Kadla, Biomacromolecules, 4, 561 (2003).

12. S. Kubo and J. F. Kadla, Macromolecules, 37, 6904 (2004).

13. S. Kubo and J. F. Kadla, J. Appl. Polym. Sci., 98, 1437 (2005).

14. S. Kubo and J. F. Kadla, Holzforschung, 60, 245 (2006).

15. J. C. Li, Y. He, and Y. Inoue, Polym. J., 33, 336 (2001).

16. Y. Li, J. Mlynar, and S. Sarkanen, J. Polym. Sci., Part B: Polym. Phys., 35, 1899 (1997).
17. Y. Li and S. Sarkanen, Macromolecules, 35, 9707 (2002).

18. Y. Li and S. Sarkanen, Macromolecules, 38, 2296 (2005).

19. W. Thielemans and R. P. Wool, Biomacromolecules, 6, 1895 (2005).

20. I. Ghosh, R. K. Jain, and W. G. Glasser, ACS Symp. Ser., 742, 331 (2000).

21. C. L. Chen, "Determination of methoxyl groups," in "Methods in lignin chemistry,” S. Y. Lin and C. W. Dence, Ed., Springer-Verlag, Berlin/Heidelberg/New York, 1992, p 465.

22. M. Y. Balakshin, E. A. Capanema, C. L. Chen, and H. S. Gracz, J. Agric. Food Chem., 51, 6116 (2003).

23. P. M. Froass, A. J. Ragauskas, and J. E. Jiang, Holzforschung, 52, 385 (1998).

24. R. H. Marchessault, S. Coulombe, H. Morikawa, and D. Robert, Can. J. Chem.-Rev. Can. Chim., 60, 2372 (1982).

25. E. Tiainen, T. Drakenberg, T. Tamminen, K. Kataja, and A. Hase, Holzforschung, 53, 529 (1999).

26. D. S. Kaplan, J. Appl. Polym. Sci., 20, 2615 (1976).

27. L. A. Utracki, "Polymer Alloys and Blends," Hanser, Munich, 1990.

28. Y. Nishio, "Cellulose Polymers, Blends, and Composites," R. D. Gilbert, Ed., Hanser, Munich/New York, 1994, chap. 5.

29. Y. Miyashita, Y. Yamada, N. Kimura, H. Suzuki, M. Iwata, and Y. Nishio, Polymer, 38, 6181 (1997).

30. M. Avella and E. Martuscelli, Polymer, 29, 1731 (1988).

31. H. D. Keith, F. J. Padden, and T. P. Russell, Macromolecules, 22, 666 (1989).

32. T. Nishi and T. T. Wang, Macromolecules, 8, 909 (1975)

33. K. Schulze, J. Kressler, and H. W. Kammer, Polymer, 34, 3704 (1993).

34. S. Nojima, D. J. Wang, and T. Ashida, Polym. J., 23, 1473 (1991).

35. J. Xu, B. H. Guo, G. Q. Chen, and Z. M. Zhang, Polym. J., 35, 460 (2003).

36. Y. Teramoto and Y. Nishio, Biomacromolecules, 5, 397 (2004).

37. R. Kusumi, Y. Inoue, M. Shirakawa, Y. Miyashita, and Y. Nishio, Cellulose, 15, 1 (2008).

38. Y. Nishio, Adv. Polym. Sci., 205, 97 (2006).

39. Y. Nishio, K. Matsuda, Y. Miyashita, N. Kimura, and H. Suzuki, Cellulose, 4, 131 (1997).

40. L. E. Nielsen, "Mechanical Properties of Polymers and Composites," Marcel Dekker, New York, 1974, p303. 